\title{
The pathobiological features of gastrointestinal cancers (Review)
}

\author{
XUE YANG $^{1}$, YASUO TAKANO ${ }^{2}$ and HUA-CHUAN ZHENG ${ }^{1}$ \\ ${ }^{1}$ Department of Biochemistry and Molecular Biology and Institute of Pathology and Pathophysiology, \\ College of Basic Medicine, China Medical University, Shenyang, P.R. China; \\ ${ }^{2}$ Clinical Research Institute, Kanagawa Cancer Center, Yokohama 241-0815, Japan
}

Received September 2, 2011; Accepted December 16, 2011

DOI: $10.3892 / \mathrm{ol} .2012 .628$

\begin{abstract}
Gastrointestinal adenocarcinoma (GIA) is a common malignant disease worldwide. Its tumorigenesis and progression is a multistage process with the involvement of a multifactorial etiology. Knowledge regarding altered expression of these genes during carcinogenesis may not only provide information about the molecular events during the initiation and progression of cancer, but may also result in the discovery of biological markers for the evaluation of cancer diagnosis and prognosis. In this review, we assessed molecular markers of pathogenesis, invasion, metastasis and prognosis, such as tumor suppressor and metastasis suppressor genes, and angiogenesis, cell adhesion, cell mobility, ER stress, mucin production, threonine protein kinase and REG family protein expression, by the establishment of tissue microarray (TMA) of GIA and immunohistochemistry (IHC) by intermittent microwave irradiation and in situ hybridization (ISH). Finally, we characterized the pathobiological features of Lauren's and WHO subtypes. It was found that the aberrant and cell-specific expression of these molecules is important in the malignant transformation of gastrointestinal epithelium and subsequent progression. These molecules also underlie the histogenic mechanisms of gastric carcinoma according to Lauren's and WHO classification. The combination of TMA, IHC and ISH may be widely applied to screen for molecular markers in GIA.
\end{abstract}

\section{Contents}

1. Introduction

2. Tumor suppressor genes

3. Metastasis suppressor genes

4. Angiogenesis

5. Cell adhesion

Correspondence to: Professor Hua-chuan Zheng, Department of Biochemistry and Molecular Biology, College of Basic Medicine, China Medical University, Shenyang, P.R. China

E-mail: zheng_huachuan@hotmail.com

Key words: gastrointestinal carcinomas, tissue microarray, immunohistochemistry, in situ hybridization, pathogenesis, pathobiological behaviors, prognosis
6. Cell mobility

7. Endoplasmic reticulum stress

8. Threonine protein kinase

9. Mucin production

10. Reg family

11. Lauren's classification

12. WHO classification

13. Conclusions

\section{Introduction}

Gastrointestinal adenocarcinoma (GIA) is a common malignant disease worldwide. Despite a worldwide decline in incidence and mortality since the second half of the 20th century, gastric cancer still ranks as the fourth most common and the second most frequent cause of mortality from cancer. Gastric cancer continues to be a major health concern due to the slow decrease in incidence in Asia and high mortality from diagnosed gastric carcinomas in the West, even though sophisticated diagnostic and surgical techniques are widely applied in clinical practice (1). Colorectal cancer is one of the most common types of cancer in the world, accounting for almost $10 \%$ of all new cases of cancer. Pathological and genetic observations demonstrated that colorectal adenoma precedes the majority of colorectal adenocarcinoma and may undergo malignant transformation into adenocarcinoma (2). Tumorigenesis and progression of gastric and colorectal carcinoma is a multistage process with the involvement of a multifactorial etiology, which mainly results from gene-environment interactions. Knowledge regarding altered expression of these genes during carcinogenesis may not only provide information about the molecular events during the initiation and progression of cancer, but may also result in the discovery of biological markers for the evaluation of cancer diagnosis and prognosis, which may aid the improvement of diagnosis, treatment and prevention of malignancies.

In the studies presented in this review, we firstly established tissue microarray (TMA) using the tissue microarryer and stained the slides with hematoxylin and eosin (HE) to confirm the histological diagnosis (Fig. 1A). Although minute TMAs cannot ensure representative areas of donor specimen, we used 2-mm diameter needles, which are large enough to evaluate the morphological appearance if the representative regions are carefully selected with HE slides. Therefore, we believe that the 

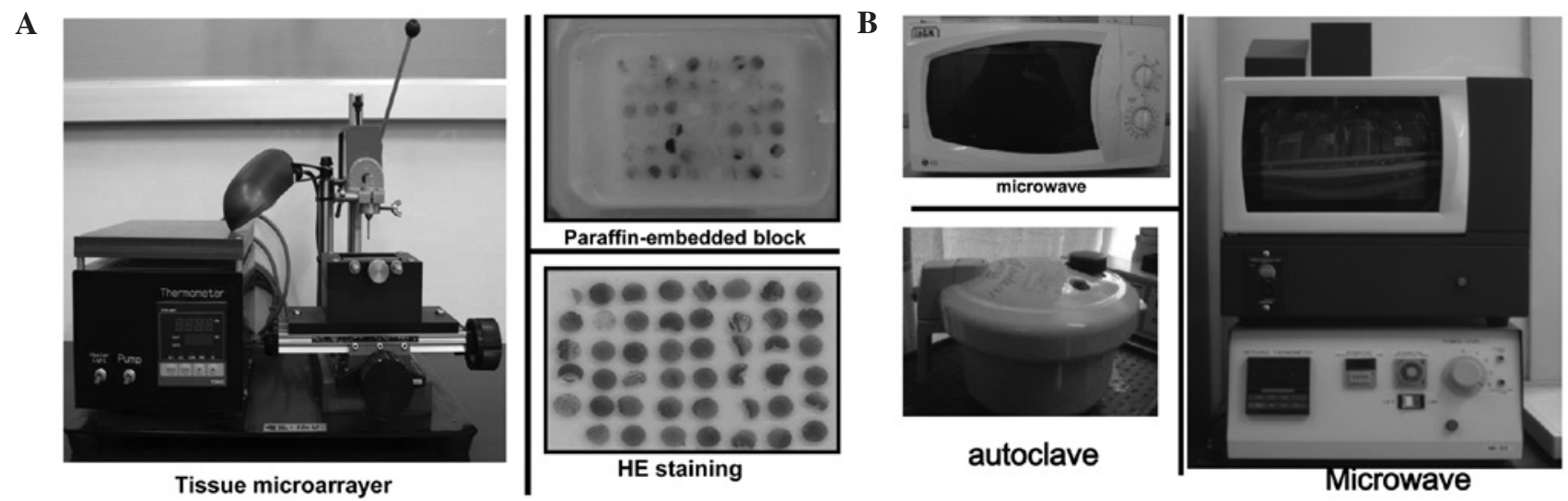

Figure 1. The combination of tissue microarray and rapid immunohistochemistry. (A) The tissue microarray was established by a tissue microarrayer and subjected to HE staining. (B) The slides were immunostained with an intermittent irradiation microwave following antigen retrieval with an electric microwave in an autoclave. HE, hematoxylin and eosin.

advantages of high throughput, identical immunohistochemical conditions, and economy of samples, antibodies and time make this approach effective for screening in clinicopathological practice (3). Additionally, a rapid immunostaining approach was employed to improve the immunoreactive quality by utilizing microwave autoclave and intermittent microwave irradiation (MI-77, Fig. 1B) during incubation. Intermittent microwaving causes minute vibrations more than 2.4 billion times/sec, which increases the probability of antibodies colliding with specific antigens. At the same time, antibodies are easily dislodged from non-specific binding sites by the motion (4). These determine the higher quality of immunohistochemistry and widen the antibodies without the application of formalin-fixed and paraffin-embedded samples in TMA (Fig. 2). Additionally, we also prepared the digoxin-labeled probes by PCR and performed the DNA-mRNA in situ hybridization (ISH) to detect the in situ expression of certain mRNA markers (Fig. 3). Using these approaches, we mainly aimed to screen for ideal markers that indicate pathogenesis, invasion, metastasis and prognosis of gastrointestinal carcinomas. The detailed findings of our previous studies (5-31) are shown in Table I and II.

\section{Tumor suppressor genes}

Malignant transformation is a biologically complicated process, resulting from frequent genetic alterations influencing the expression of oncogenes and tumor suppressor genes (TSG). Generally, chromosomal deletion may lead to loss of TSG causing uncontrolled proliferation and immortal survival, critical for initiation, promotion and tumor development.

p53 is thought to play a central role in protecting against the development of cancer. Its encoding protein is a master switch that coordinates and concentrates a plethora of stress signals and transforms them into a series of responses, such as apoptosis or cell cycle arrest in response to DNA damage, thereby maintaining genetic stability in the organism. Although p53 inactivation in human cancer is a complex process, depending on the tissue type, p53 dysfunction may disorder the biological events of cancer cells and give rise to their aggressive phenotypes. Currently, no antibody can discriminate between the mutant and wild-type p53 protein, although the anti-p53 antibody is widely applied in clinical practice. In our study, the p53 expression was gradually increased from gastrointestinal mucosa to adenoma to adenocarcinoma, and showed a positive association with depth of invasion, local invasion via vessels and lymph node metastasis of GIA, suggesting that the majority of accumulated p53 proteins may be of the mutant subtype in GIA or adenocarcinoma (5).

The PTEN gene (phosphatase and tensin homology deleted from human chromosome 10) inhibits Shc phosphorylation and therefore blocks the activation of the Ras/MAP-kinase pathway. PTEN also dephosphorylates focal adhesion kinase (FAK), affecting cell adhesion, spreading and recognition. Furthermore, PTEN acts as a phospholipid phosphatase with phosphatidylinositol 3, 4, 5-trisphosphate (PIP3) as a substrate and one down-stream target of PIP3, protein kinase (Akt/PKB), is continually activated by phosphorylation in cells lacking in functional PTEN. PTEN expression was lower in gastric carcinoma than that in non-neoplastic mucosa (NNM), adenoma or carcinoma of the stomach and colorectum, and inversely correlated with tumor size, depth of invasion, lymphatic invasion, venous invasion, lymph node metastasis, TNM staging, lower caspase-3 expression, and worse prognosis of gastric and colorectal carcinoma. The mutation analysis revealed only one synonymous mutation in exon 8 (codon 312 Asp: GAC $\rightarrow$ GAT) in colorectal carcinoma using high fidelity polymerase and direct DNA sequencing. In our studies, the anti-PTEN antibody can recognize the nuclear protein, although PTEN functions as a phosphatase in the cytosol. PTEN has nuclear localization signal-like sequences for nuclear import mediated by a major vault protein and is required for cell cycle arrest in the nucleus $(6,7)$.

FHIT (fragile histine triad) was isolated by positional cloning, and encompassed the most common human fragile site FRA3B at 3p14.2, a region with frequent hemizygous and homozygous deletion in a variety of human tumors. Studies on protein-protein interactions, cell lines, tumorigenicity tests and knockout mice suggest that the FHIT protein is involved in cell proliferation and apoptosis, and may act as a tumor suppressor independent of its hydrolase activity. FHIT was less expressed in gastric NNM and adenoma than gastritis. FHIT expression showed a significantly negative association with depth of invasion, lymphatic invasion, lymph node metastasis, liver metastasis, UICC staging and worse prognosis of 

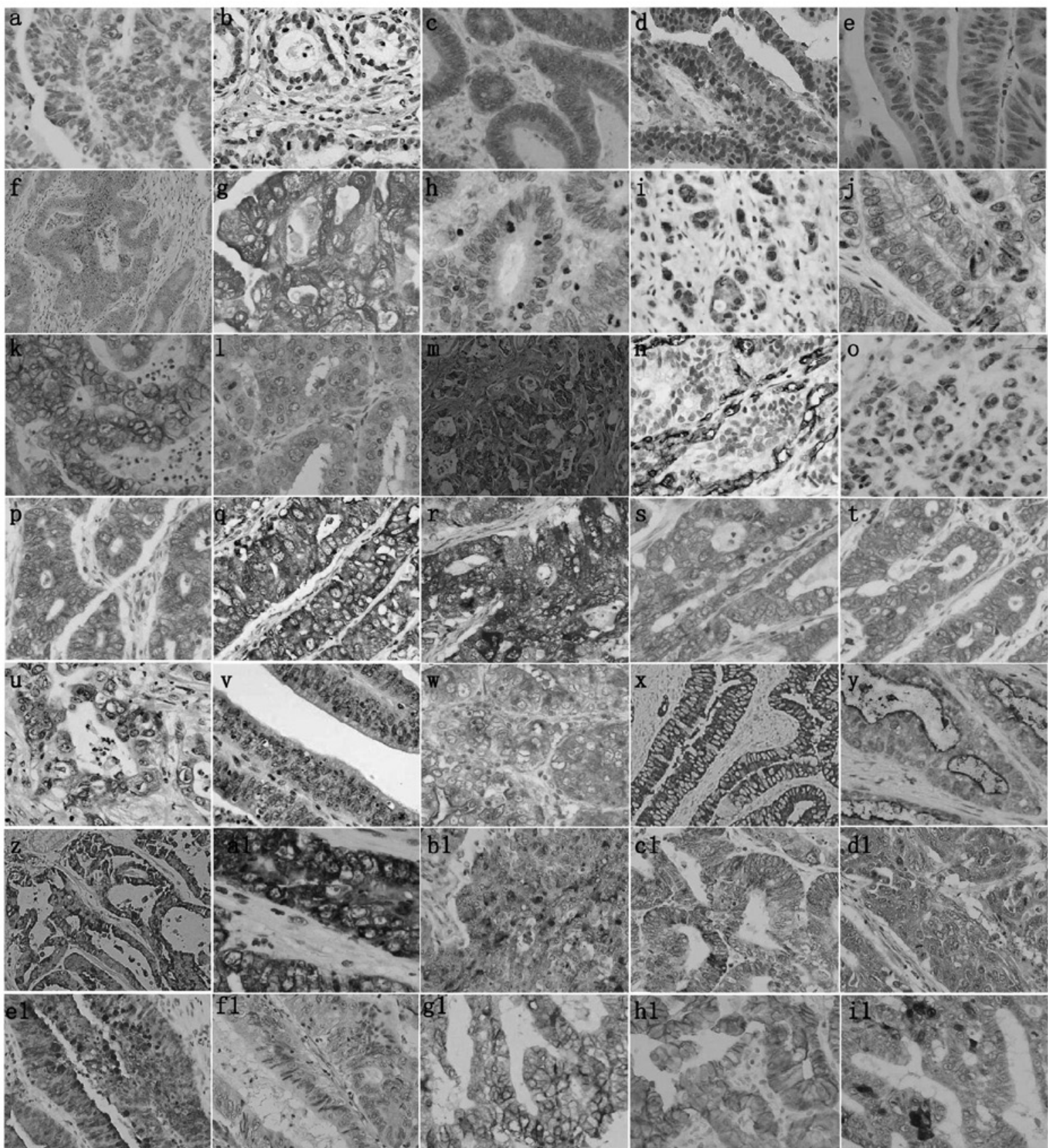

Figure 2. Immunohistochemical staining of various markers in gastrointestinal carcinomas. (a) p53, (b) PTEN, (c) FHIT, (d) ING5, (e) Parafibromin, (f) KAI1, (g) maspin, (h) MMP-2, (i) MMP-7, (j) MMP-9, (k) EMMPRIN, (l) VEGF, (m) tenascin, (n) CD34, (o) Arp2, (p) Arp3, (q) cortactin, (r) fascin, (s) GRP78, (t) RP94, (u) GSK3 $\beta-$ ser $^{9}$, (v) Pim-3, (w) MUC-1, (x) MUC-2, (y) MUC-4, (z) MUC-5AC, (a1) MUC-6, (b1) REG I $\alpha$ (c1) REG I $\beta$, (d1) REGIII, (e1) HIP/PAP, (f1) REG IV, (g1) CD44, (h1) E-cadherin, (i1) $\beta$-catenin.

gastric carcinoma, but its functions have not been sufficiently explored (6).

Inhibitor of growth 5 (ING5) interacts with histone H3K4me 3 and is involved in the formation of two different histone acetyl transferase (HAT) complexes, which have an important role during DNA replication in cooperation with the mini-chromosome maintenance complex. ING5 was reported to activate the cyclin-dependent kinase inhibitor $p 21 /$ wafl promoter to induce $\mathrm{p} 21 / \mathrm{waf} 1$ expression, enhance p53 acetylation at Lys-382 residues, and physically interact with p300, a member of the HAT complexes, and p53 in vivo. In our GIAs, ING5 protein has nuclear-cytoplasmic translocation with aggressive transformation in the colorectal adenoma-adeno- carcinoma sequence. Downregulated nuclear ING5 expression may be employed to indicate worse behaviors or prognosis, while it was the converse for the cytoplasmic counterpart in the two carcinomas. The subcellular distribution of ING5, its biological effects on cell phenotypes and related molecular mechanisms should be further investigated in the future $(8,9)$.

Parafibromin is be involved in the formation of the Paf1 complex, which is associated with RNA polymerase II and involved in transcript site selection, transcriptional elongation, histone $\mathrm{H} 2 \mathrm{~B}$ ubiquitination, histone $\mathrm{H} 3$ methylation, poly (A) length control, and coupling of transcriptional and post-transcriptional events. Parafibromin overexpression was found to inhibit colony formation and cellular proliferation and induce 


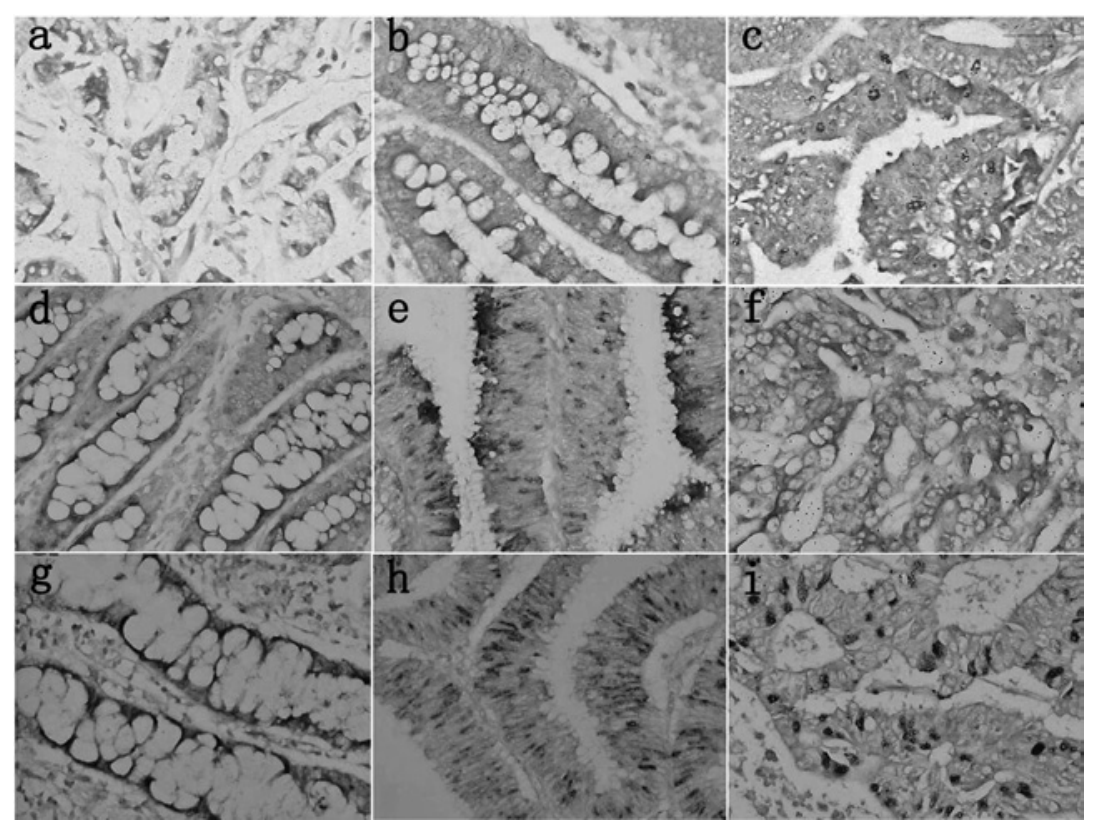

Figure 3. In situ hybridization on TMA of gastrointestinal carcinoma. REG IV mRNA positivity was observed in (a) gastric mucosa, (b) intestinal metaplasia and (c) carcinoma. There was EMMPRIN and parafibromin mRNA expression in (d and g) colorectal mucosa, (e and h) adenoma and (f and i) carcinoma, respectively. TMA, tissue microarray.

cell cycle arrest in the G1 phase, indicating that parafibromin has a critical role in cell growth. RNA interference with the expression of parafibromin or Paf1 was proved to stimulate cell proliferation and increase the c-myc level by stabilizing c-myc protein and activating the c-myc promoter. We found that parafibromin expression was gradually decreased from gastric and colorectal carcinogenesis regardless of protein or mRNA levels. Its downregulated expression was inversely correlated with tumor size, depth of invasion, lymphatic invasion, lymph node metastasis, UICC staging or shorter survival time. In addition, parafibromin overexpression caused G1 arrest and enhanced differentiation of DLD-1 cells. A high expression of p21, p27 and cyclin E, but a low expression of cyclin D1 mRNA, phospho-cdc2 and phospho-cdc25c proteins was observed in parafibromin-overexpressing DLD-1. Parafibromin inhibited $c$-myc mRNA expression by binding to the $c-m y c$ promoter in colorectal carcinoma cells. The signal pathways of nuclear parafibromin are being established by phenotype, protein-protein or protein-DNA analyses in GIAs $(10,11)$.

\section{Metastasis suppressor genes}

KAI1 (CD82/C33/R2/IA4) was initially identified as a tumor metastasis suppressor gene and encodes transmembrane glycoproteins of the tetraspanins family (TM4SF). TM4SF proteins have cytoplasmic $\mathrm{N}$ - and $\mathrm{C}$-termini and traverse the cell membrane four times forming one small and one large extracellular loop with residues susceptible to post-translational phosphorylation and/or N-linked glycosylation. KAI1 interacts with integrin $\alpha 4 \beta 1$, other TM4SF proteins, and cell surface molecules including CD4, CD8, CD19, CD21 and MHC class I and II, forming 'the tetraspanin web'. Several recent reports suggested that a complex combination between KAI1 and specific proteins is involved in a number of biological processes, such as cellular adhesion, mobility, proliferation and apoptosis $(12,13)$. In our study, KAI1 expression was higher in GIAs than that in their adjacent NNM and showed a significantly negative association with the liver metastasis of GIAs, which is useful and may aid in predicting the risk of liver metastasis (14).

Maspin exhibits significant homology to the super-family of such serine protease inhibitors as the plasminogen activator inhibitors 1 and 2, and $\alpha 1$-antitrypsin, which is closely linked to the degradation of extracellular matrix (ECM). Maspin has been shown to inhibit tumor cell motility and invasion in mammary and prostate carcinoma cell lines in vitro, and to reduce the capacity for tumorigenesis and metastasis of cancer cell lines in animal models. However, in vivo experiments have indicated a decrease and an increase of maspin levels in tumors, and levels of the protein have paradoxically been described to parallel tumor progression in spite of being regarded as a tumor suppressor gene (15-19). In this study, it was also found that cytoplasmic and nuclear maspin expression paralleled each other and decreased from gastric intestinal metaplasia, adenoma and carcinoma to normal mucosa. A significant positive association was noted with depth of invasion, lymphatic invasion, lymph node metastasis, TNM stage and cumulative patient survival in gastric carcinoma. By contrast, maspin expression showed a significant increase from colorectal NNM to adenocarcinoma through adenoma, and correlated negatively with the liver metastasis of colorectal carcinomas. Therefore, the tissue specificity of maspin and its function in the nucleus should be studied in future investigations $(20,21)$.

\section{Angiogenesis}

Growth of solid tumors depends on angiogenesis, which facilitates metastasis formation, and provides nutrients and oxygen for growth at the metastasis site. Critical steps during tumor angiogenesis are the outgrowth of endothelial cells from 
Table I. The protein expression in gastrointestinal carcinogenesis.

\begin{tabular}{lll}
\hline Groups & \multicolumn{1}{c}{ Up-regulated molecules } & Down-regulated molecules \\
\hline Intestinal metaplasia & Maspin, EMMPRIN, Arp2 & - \\
Adenoma & p53, maspin, EMMPRIN, fascin, GRP78, GRP94, & PTEN, parafibromin \\
& Pim-3, MUC-6, REG IV & \\
Carcinoma & p53, KAI1, maspin, EMMPRIN, Arp2, Arp3, & PTEN, FHIT, ING5, parafibromin, \\
& cortactin, fascin, GRP78, GRP94, GSK3 $\beta$-ser 9 , Pim-3, & MUC-6, REG I $\beta$
\end{tabular}

Compared with non-neoplastic mucosa or gastritis. EMMPRIN, extracellular matrix metalloproteinase inducer; Arp, actin-related protein; PTEN, phosphatase and tensin homology deleted from human chromosome 10; FHIT, fragile histine triad; ING5, inhibitor of growth 5; HIP/PAP, hepatocellular carcinoma-intestine-pancreas gene/pancreatitis-associated protein.

preexisting capillary vessels and their migration from parental vessels under the stimulation of vascular endothelial growth factor (VEGF). Proliferating endothelial cells subsequently remodel ECM via matrix metalloproteinases (MMPs), align into tube-like structures, and eventually form new functional blood vessels. In cancer cells, overexpression of angiogenic factors (e.g., VEGF and MMPs) results in their increased secretion into the ECM to stimulate the proliferation and mobility of vascular epithelial cells, closely linked to invasion and metastasis.

MMPs are a family of enzymes that proteolytically degrade various components of the ECM. A high expression of MMPs by the tumor cells is closely correlated with tumor invasive and metastatic potential. MMP-2 (72 kDa), -7 (19 kDa) and -9 $(92 \mathrm{kDa})$ have been shown to play critical roles in the 'angiogenic switch' and tumor cells synthesize and secret large amounts of MMP-2 and -9 in a paracrine and/or autocrine manner to stimulate angiogenesis and increase VEGF release. VEGF is a $45-\mathrm{kDa}$ homodimeric glycoprotein, which acts as a potent and selective endothelial mitogen. Our group found that the expression of MMP-2, -7, -9 and VEGF were positively correlated with tumor size, depth of invasion, lymphatic and venous invasion, lymph node metastasis, TNM staging and microvessel density (MVD) of gastric carcinomas. VEGF expression was also positively correlated with the levels of MMP-2 and -9, but negatively with PTEN. The latter was also inversely associated with the MVD in gastric carcinomas $(22,23)$.

The extracellular region of EMMPRIN (extracellular matrix metalloproteinase inducer) contains three $\mathrm{N}$-linked glycosylation sites, and this extracellular region is responsible for the MMP-stimulating activity. EMMPRIN is expressed in highly- (HG 45-65 kDa), poorly-glycosylated (LG 32-44 kDa) and core protein (approximately $27 \mathrm{kDa}$ ) forms. EMMPRIN was reported to stimulate tumor angiogenesis by elevating VEGF and MMP expression in neighboring fibroblasts and epithelial cells. In our study, it was found that EMMPRIN expression was gradually increased from normal mucosa to carcinomas through hyperplastic or metaplastic mucosa of the stomach, and positively correlated with tumor size, depth of invasion, lymphatic invasion, expression of ki-67, MMP-2, -9 and VEGF, MVD and poor prognosis of gastric carcinoma. In colorectal carcinoma, EMMPRIN expression was immunohistochemically stronger in colorectal high-grade adenoma, adenocarcinoma and metastatic carcinoma compared to nonneoplastic superficial epithelium and low-grade adenoma, and positively correlated with tumor size, depth of invasion, vascular or lymphatic invasion, grade of infiltration and VEGF expression of carcinomas. However, the manner in which these angiogenesis-related factors regulate each other at the transcriptional level is likely to become a crucial issue as the hydrolytic effects of MMPs on transmembrane proteins are clarified $(24,25)$.

\section{Cell adhesion}

Tenascin is a large ECM glycoprotein with a six-armed disulfide-bonded macromolecular structure, consisting of tenascin- $\mathrm{C}$ (formerly known under various synonyms, such as cytotactin and hexabrachion), tenascin-R (for restrictin), -X, -Y and -W. All family members share a modular structure, consisting of a cysteine-rich $\mathrm{N}$-terminal domain involved in the oligomerization of tenascin- $\mathrm{C},-\mathrm{R}$, and possibly $-\mathrm{X}$, as well as a series of epidermal growth factor-like repeats, followed by a number of fibronectin type III-like domains and a carboxy-terminal fibrinogen-like domain. Tenascin has a number of biological functions likely to affect tumor development, such as the regulation of tumor cell-cell interaction, proliferation, invasion and metastasis, and involvement in angiogenesis. Tenascin expression showed a significantly negative association with liver metastasis of GIA. There was a significantly negative relationship between EMMPRIN and tenascin expression in GIA, indicating that EMMPRIN induced the production of MMPs, which degrade the tenascin (14).

\section{Cell mobility}

The aggressive phenotype depends on cell adhesiveness, motility and deformability, which are thought to result from quantitative alterations and the rearrangement of various cytoskeletal components, disassembly of actin filaments and actin polymerization. The cytoskeleton is composed of actin filaments, intermediate filaments and microtubules. In particular, actin-binding proteins such as cortactin and fascin are involved in the cytoskeleton formation, cell migration and signaling pathways. The actin-related protein (Arp)2/3 complex has been identified to bind to itself and directly regulates the actin 
Table II. The relationship between the protein expression and clinicopathological parameters of gastrointestinal carcinomas.

Groups $\quad$ Upregulated molecules $\quad$ Downregulated molecules

Age (young)

Gender (Male)

Tumor size (small)

Depth of invasion

(superficial)

Lymphatic invasion (+)

Venous invasion $(+)$

Lymph node metastasis (+) PTEN, FHIT, nuclear ING5, parafibromin,

Liver metastasis

UICC staging (low)

Lauren's classification (Intestinal)

Favorable prognosis
PTEN, FHIT, parafibromin, MUC-6

FHIT, MUC-6, REG I $\beta$, HIP/PAP MUC-6, REG I $\alpha$, HIP/PAP

PTEN,FHIT, nuclear ING5, parafibromin, MUC-6

\author{
Cortactin, GRP94, GSK3 $\beta$-ser ${ }^{9}$, Pim-3, FHIT
}

Nuclear ING5, parafibromin, MUC-6

PTEN, FHIT, nuclear ING5, parafibromin, MUC-6, REG I $\alpha$, REG I $\beta$, REG III, HIP/PAP

p53, FHIT, nuclear ING5, parafibromin, maspin, MMP-2, MMP-7, MMP-9,

EMMPRIN, VEGF, cortactin, Pim-3, MUC-1, MUC-6, VEGF, GSK3 $\beta$-ser ${ }^{9}$, MUC-2,

MUC- 4, CD44, E-cadherin,

membrane $\beta$-catenin

PTEN, FHIT, nuclear ING5, parafibromin, MUC-6
Nuclear ING5, parafibromin, maspin, fascin, GRP94

MMP-2, MMP-7, MMP-9, EMMPRIN, VEGF, Arp2, Arp3, cortactin, fascin, GRP78, GRP94

p53, cytoplasmic ING5, maspin, MMP-2, MMP-7, MMP-9, EMMPRIN, VEGF, Arp2, Arp3, cortactin, fascin, GRP78, GRP94, GSK3 $\beta$-ser ${ }^{9}$, MUC-1

p53, maspin, MMP-2, MMP-9, EMMPRIN, VEGF, cortactin, fascin, GRP78, GRP94, GSK3 3 -ser ${ }^{9}$, Pim-3, MUC-1

p53, cytoplasmic ING5, MMP-2, MMP-9, EMMPRIN, VEGF, Arp2, Arp3, cortactin, fascin, GRP78, GRP94, GSK3 $\beta$-ser ${ }^{9}$,

Pim-3, MUC-1

p53, cytoplasmic ING5, maspin, MMP-2, MMP-7, MMP-9, VEGF, cortactin, fascin, GRP78, GRP94, GSK3 $\beta$-ser ${ }^{9}$, MUC-1

PTEN, FHIT, KAI1, tenascin

cytoplasmic ING5, maspin, MMP-2, MMP-7, MMP-9, VEGF, Arp2, Arp3, cortactin, fascin, GRP78, GRP94, MUC-1

Cytoplasmic ING5

Maspin, EMMPRIN, fascin, GRP78, GRP94, GSK3 $\beta$-ser ${ }^{9}$, Pim-3, MUC-1

ING5, inhibitor of growth 5; FHIT, fragile histine triad; MMP, matrix metalloproteinase; EMMPRIN, extracellular matrix metalloproteinase inducer; VEGF, vascular endothelial growth factor; Arp, actin-related protein; HIP/PAP, hepatocellular carcinoma-intestine-pancreas gene/ pancreatitis-associated protein; PTEN, phosphatase and tensin homology deleted from human chromosome 10; UICC, Union for International Cancer Control.

polymerization reaction by interacting with Wiskott-Aldrich syndrome-related protein family proteins. Cortactin is a filamentous actin-binding monomer and consists of an aminoterminal acidic (NTA) region, 37-residue-long segments, a proline-rich region and an SH3 domain. The NTA region harbors a short motif called DDW, which is necessary for binding to the Arp2/3 complex. Arp2 and -3 closely resemble the structure of monomeric actin and serve as nucleation sites for new actin filaments to stimulate actin polymerization. In vitro experimental evidence indicated that fascin overexpression correlated with increased proliferation, altered $\beta 1$ integrin distribution, enhanced the invasive capacity and altered the differentiation status in colonic adenocarcinoma.

The expression of cortactin and fascin was higher in gastric carcinomas than adenoma and NNMs, and positively correlated with tumor size, depth of invasion, lymphatic and venous invasion, lymph node metastasis and TNM staging. Arp2 and -3 proteins were expressed at low levels in gastritis, compared with carcinomas. Arp2 was more frequently expressed in intestinal metaplasia than in carcinoma or gastritis. Arp 2 and -3 proteins were positively correlated with tumor size, depth of invasion, venous invasion, UICC staging and expression of cortactin or fascin. Univariate analysis indicated that the cumulative survival rate of patients with positive fascin expression was lower than that without its expression, even when stratified according to the depth of invasion. Lamellipodia and invadopodia formation of gastric carcinoma cells have yet to be adequately investigated. If the effects of these mobility-related proteins on invasion and migration are clarified, the anti-metastatic therapy is likely 
to be improved for patients with advanced gastrointestinal carcinomas $(26,27)$.

\section{Endoplasmic reticulum stress}

The endoplasmic reticulum (ER) is important in regulating the synthesis, folding and targeting of secretory and membrane proteins. Oxidative stress, glucose deprivation, chemical toxicity, alterations in intracellular $\mathrm{Ca}^{2+}$ levels, blockade of glycosylation and hypoxia induce ER stress, in which the expression of glucose-related proteins (GRPs) is activated. GRPs are ubiquitously expressed in ER and is capable of assisting in protein folding and assembly, and are consequently considered as molecular chaperones.

There was more expression of the two proteins in gastric carcinoma and adenoma than in NNM. The two proteins were positively correlated with tumor size, depth of invasion, lymphatic and venous invasion, lymph node metastasis, TNM staging and worse prognosis of gastric carcinoma. In colorectal carcinoma, there was a gradually increased GRP78 expression from NNMs and carcinomas, to low-grade and high-grade adenomas, while up-regulated GRP94 expression occurred from NNM, low-grade adenoma and high-grade adenoma to carcinoma. GRP78 expression was negatively correlated with lymphatic invasion or a low GRP94 expression of colorectal carcinomas. The significance of GRPs in gastric and colorectal carcinoma may be explained by the distinct histogenesis and behaviors of the two carcinomas $(28,29)$.

\section{Threonine protein kinase}

Glycogen synthase kinase-3 $\beta$ (GSK3 $\beta$ ) is a serine/threonine protein kinase, which may be involved in protein synthesis, cell proliferation, cell differentiation, microtubule dynamics and cell motility by phosphorylating initiation factors, components of the cell-division cycle, transcription factors and proteins involved in microtubule function and cell adhesion. The activity of GSK $3 \beta$ is inhibited via ser- 9 phosphorylation by p70 S6 kinase, p90Rsk, Akt, certain isoforms of protein kinase, and cyclic AMP-dependent protein kinase. Inactive GSK3 $\beta$ phosphorylated at ser-9 was more expressed in gastric carcinomas than in NNM, and positively correlated with depth of invasion, lymphatic and venous invasion, lymph node metastasis, TNM staging, expression of VEGF and EMMPRIN, and poor prognosis in gastric carcinoma (30).

Pim-3, a member of the proto-oncogene Pim family with serine/threonine kinase activity, was aberrantly expressed in cancer lesions of endoderm-derived organs. The ablation of Pim-3 expression induced the apoptosis of human hepatocellular, pancreas and colon carcinoma cell lines. Moreover, Pim-3 can inactivate a potent pro-apoptotic molecule, Bad, in human pancreas and colon carcinoma cells, by phosphorylating its Ser112, thereby preventing apoptosis. Pim-3 expression was enhanced in adenoma and metastasis sites of gastric carcinoma and, to a lesser degree, in primary sites of gastric carcinoma, compared with NNM. Pim-3 expression was positively correlated with lymphatic and venous invasion, a high expression of VEGF and EMMPRIN, a low PTEN expression and poor prognosis. However, the biological functions of Pim-3 should be further studied in gastric carcinoma, particularly regarding differentiating induction (31).

\section{Mucin production}

The mucin components of the gastric gel layer function as a protective and lubricating factor against luminal acid and proteolytic enzymes, and also hinder access of carcinogens causing DNA damage. When the stomach suffers from infection with Helicobacter pylori (HP), a group I carcinogen, HP lipopolysaccharides decrease mucin synthesis by the phosphatidylinositol 3-kinase/ERK pathway and via the inhibition of galactosyltransferase.

In malignancies, MUC-1 may function as an anti-adhesion molecule facilitating the release of cells from tumor nests and may mask extracellular domains of cancer cells from immune surveillance. MUC-6 is essential in epithelial cytoprotection against a wide range of substances. In gastric carcinoma, MUC-1 was found to be highly expressed in gastric carcinomas in comparison with NNM and positively correlated with depth of invasion, lymphatic and venous invasion, lymph node metastasis, TNM staging, poor prognosis, and MUC-4 expression, while it was the converse for MUC-6. It is of great significance to characterize the mucin production of gastric carcinoma, particularly in signet ring cell carcinoma (SCR) $(32,33)$.

\section{Reg family}

The Regenerating (Reg) gene family belongs to the calcium-dependent lectin (C-type lectin) gene superfamily, which encodes a group of small multifunctional secretory proteins. Reg family proteins function as acute phase reactants, lectins, anti-apoptotic factors and growth agents. These proteins are primarily involved in cell proliferation and differentiation, inflammation, diabetes and carcinogenesis. Three subtypes of $R E G$ gene have been identified in humans, including REG I (I $\alpha$ and I $\beta$ ), REG III (III and HIP/PAP: hepatocellular carcinomaintestine-pancreas gene/pancreatitis-associated protein) and $R E G I V$. REG IV has been reported to be a potent activator of the epidermal growth factor receptor/Akt/activator protein-1 signaling pathway in colon cancer cells and increases the expression of BCL-2, Bcl-xL, and survivin proteins. REG IV treatment protects normal intestinal crypt cells from irradiation-induced apoptosis by increasing the expression of BCL-2, Bcl-xL and survivin.

Our group found that REG IV protein expression was gradually decreased from gastric IM, adenoma and carcinoma to gastritis. The positive rate of its mRNA was higher in gastric IM than carcinoma or NNM. Reg IV expression was significantly correlated with the MUC-2 and MUC-5AC expression. In colorectal carcinoma, the expression of REG I $\alpha$, III and HIP/ PAP was more frequently observed in colorectal carcinomas than in adjacent NNM, while it was the converse for REG I $\beta$ and IV. The expression of REG I $\alpha$, I $\beta$, III and HIP/PAP was negatively correlated with the depth of invasion of CRCs. REG I $\beta$ and HIP/PAP were less expressed in CRCs with than without venous invasion. The positive rates of REG I $\alpha$ and HIP/PAP were significantly higher in CRCs without than with lymph node metastasis. A positive relationship was found between REG I $\alpha$, I $\beta$, III and HIP/PAP expression. Survival analysis indicated that 
the REG I $\beta$ or HIP/PAP expression was positively correlated with favorable prognosis of carcinoma patients. To the best of our knowledge, the receptors and biological functions of REG proteins remain to be clarified $(34,35)$.

\section{Lauren's classification}

Lauren's classification has been widely applied in clinical practice and is useful in clarifying the histopathogenesis with epidemiological priority. Histologically, the intestinal types principally include papillary, well-differentiated, moderatelydifferentiated or mucinous adenocarcinoma without SCR cells, whereas the diffuse-type mainly consisted of the poorly-differentiated adenocarcinoma, SRC carcinoma and undifferentiated adenocarcinoma of the World Health Organization (WHO) classification. Although approximately $15 \%$ of gastric carcinomas are characterized as unclassified or mixed type, an intermediate type of gastric carcinoma may show a few special changes, reflecting polycolonal histogenesis and aggressiveness. Our group found that intestinal-type carcinoma frequently occurred in older males, while comparatively, the diffuse-type occurred in young women. The latter was more inclined to invasion into muscularis propria, lymphatic invasion and lymph node metastasis, and belonged to higher TNM staging, compared with intestinal-type counterparts. Mixed-type (MT) carcinomas exhibited large size, deep invasion, frequent local invasion and lymph node metastasis in comparison with intestinal- and diffuse-type carcinoma. Nuclear ING5, cortactin, MUC-1, MUC-6, parafibromin, Pim-3, p53, FHIT, maspin, VEGF, GSK3 $\beta$-ser ${ }^{9}$, MUC-2, MUC-4, CD44, E-cadherin, membrane $\beta$-catenin and EMMPRIN showed a higher expression in intestinal- than diffuse-type carcinomas. The expression of maspin, EMMPRIN, VEGF, MUC-4 and membrane E-cadherin was stronger in MT intestinal than diffuse components. Immunoreactivities to EMMPRIN and VEGF were weaker in intestinal carcinoma than in the MT intestinal portion, whereas the opposite was true for CD44, MUC-2 and -6. The MT diffuse component exhibited a higher expression of FHIT, VEGF and P-GSK3 $\beta$-ser ${ }^{9}$ than diffuse-type carcinoma. These findings suggested that MT carcinomas were also shown to be more aggressive and that different components of mixed-type carcinoma may originate from common stem cells, but follow a distinct histogenic pathway, as the significant difference in the proliferation, apoptosis, angiogenesis, mucin secretion and cell adhesion between the intestinal- and diffuse-type carcinomas becomes smaller between intestinal and diffuse components of the MT carcinoma $(36,37)$.

\section{WHO classification}

The WHO classification classifies adenocarcinoma into papillary, well-, moderately-, or poorly-differentiated, mucinous adenocarcinoma, SRC and undifferentiated carcinoma. WHO classification is performed according to histomorphological features and it is easy to establish its relationship with other grouping approaches, including Lauren's, Nakamura and Goseki's classification. The majority of cases were well-, poorly-, or moderately-differentiated subtypes, whereas the minority were papillary or SRC. Patients with poorly-differentiated or SRC carcinoma were predominantly young and female. Poorly-differentiated and mucinous carcinomas were larger, with deeper invasion, more venous or lymphatic invasion, frequent lymph node involvement and peritoneal dissemination, or higher staging. The SRC group exhibited a weaker expression of caspase-3, p53, parafibromin, GRP78, GRP94, P-GSK3 $\beta-$ ser $^{9}$, VEGF or cortactin. The moderatelydifferentiated subtype exhibited a lower expression of FHIT and Arp3 positivity. The poorly-differentiated group showed a weaker expression of caspase-3, EMMPRIN, MUC-2, MUC-5AC and MUC-6. Mucinous carcinoma more frequently expressed REG IV protein than well-, and moderatelydifferentiated carcinomas. Survival analysis indicated that the patients with poorly-differentiated or mucinous subtypes had a lower cumulative survival rate than those with papillary, well-, moderately-differentiated or SRC carcinomas $(34,38)$.

\section{Conclusions}

The aberrant and cell-specific expression of molecules such as tumor suppressor and metastasis suppressor genes, angiogenesis, cell adhesion, cell mobility, ER stress, mucin production, threonine protein kinase and REG family proteins is essential in the malignant transformation of gastrointestinal epithelium and subsequent cancer development. This expression also underlies the histogenic mechanisms of gastric carcinoma according to Lauren's and WHO classification. In MT carcinomas, the different components of mixed-type carcinoma may originate from common stem cells, but follow distinct histogenic pathways. Protein-protein or protein-DNA interaction may be combined with phenotype analysis to establish the pathways of cytoplasmic or nuclear proteins in GIAs. Additionally, morphological examination of certain markers may generate some novel questions. For instance, the in vivo effects of certain molecules are occasionally opposite to their in vitro functions, which requires further investigation. Additionally, lamellipodia and invadopodia formation of gastric or colorectal carcinoma cells has not been fully investigated, with the effects of these mobility-related proteins on invasion and migration remaining to be clarified, which has blocked the anti-metastatic therapy of advanced GIAs. According to histological classification, SRC is of note, posing the question of whether there is a possible relationship between cell adhesion and mucin synthesis in its histogenesis. In this review, we only observed the in situ expression of well-known genes or proteins, which is a limitation of the review. Additionally, the sensitivity and specificity of the IHC and ISH are dependent on the antibody or probe, and are also determined by the tissue contents of the protein and mRNA. The combination of TMA, IHC and ISH may therefore be widely applied to screen molecular markers in GIA.

\section{Acknowledgements}

This study was supported by the Shenyang Outstanding Talent Foundation of China; Shenyang Science and Technology Grant (1091175-1-00; F11-264-1-10); Liaoning Science and Technology Grant; Natural Scientific Foundation of China (No. 81001093; No. 81101885; No. 81101886; No. 81172371); Grantin-aid for Scientific Research from the Ministry of Education, Culture, Sports and Technology of Japan (23659958). 


\section{References}

1. Kelley JR and Duggan JM: Gastric cancer epidemiology and risk factors. J Clin Epidemiol 56: 1-9, 2003.

2. American Cancer Society. Colorectal cancer facts and figures 2005.

3. Moch H, Schraml P, Bubendorf L, Mirlacher M, Kononen J, Gasser T, Mihatsch MJ, Kallioniemi OP and Sauter G: High-throughput tissue microarray analysis to evaluate genes uncovered by cDNA microarray screening in renal cell carcinoma. Am J Pathol 154: 981-986, 1999.

4. Kumada T, Tsuneyama K, Hatta H, Ishizawa S and Takano Y: Improved 1-h rapid immunostaining method using intermittent microwave irradiation: practicability based on 5 years application in Toyama Medical and Pharmaceutical University Hospital. Mod Pathol 17: 1141-1149, 2004.

5. Zheng H, Tsuneyama K, Cheng C, Takahashi H, Cui Z, Murai Y, Nomoto K and Takano Y: An immunohistochemical study of P53 and $\mathrm{Ki}-67$ in gastrointestinal adenoma and adenocarcinoma using tissue microarray. Anticancer Res 26: 2353-2360, 2006.

6. Zheng H, Takahashi H, Murai Y, Cui Z, Nomoto K, Tsuneyama K and Takano Y: Low expression of FHIT and PTEN correlates with malignancy of gastric carcinomas: tissue-array findings. Appl Immunohistochem Mol Morphol 15: 432-440, 2007.

7. Li XH, Zheng HC, Takahashi H, Masuda S, Yang XH and Takano Y: PTEN expression and mutation in colorectal carcinomas. Oncol Rep 22: 757-764, 2009.

8. Xing YN, Yang X, Xu XY, Zheng Y, Xu HM, Takano Y and Zheng HC: The altered expression of ING5 protein is involved in gastric carcinogenesis and subsequent progression. Hum Pathol 42: 25-35, 2011.

9. Zheng HC, Xia P, Xu XY, Takahashi $\mathrm{H}$ and Takano Y: The nuclear to cytoplasmic shift of ING5 protein during colorectal carcinogenesis with their distinct links to pathologic behaviors of carcinomas. Hum Pathol 42: 424-433, 2011.

10. Zheng HC, Takahashi H, Li XH, Hara T, Masuda S, Guan YF and Takano Y: Downregulated parafibromin expression is a promising marker for pathogenesis, invasion, metastasis and prognosis of gastric carcinomas. Virchows Arch 452: 147-155, 2008

11. Zheng HC, Wei ZL, Xu XY, Nie XC, Yang X, Takahashi H and Takano Y: Parafibromin expression is an independent prognostic factor for colorectal carcinomas. Hum Pathol 8: 1089-1102, 2011.

12. Lee JH, Park SR, Chay KO, Seo YW, Kook H, Ahn KY, Kim YJ and Kim KK: KAI1 COOH-terminal interacting tetraspanin (KITENIN), a member of the tetraspanin family, interacts with KAI1, a tumour metastasis suppressor, and enhances metastasis of cancer. Cancer Res 64: 4235-4243, 2004.

13. Schoenfeld N, Bauer MK and Grimm S: The metastasis suppressor gene C33/CD82/KAI1 induces apoptosis through reactive oxygen intermediates. FASEB J 18: 158-160, 2004.

14. Zheng H, Tsuneyama K, Cheng C, Takahashi H, Cui Z, Nomoto K, Murai Y and Takano Y: Expression of KAI1 and tenascin, and microvessel density are closely correlated with liver metastasis of gastrointestinal adenocarcinoma. J Clin Pathol 60: 50-56, 2007.

15. Maass N, Hojo T, Ueding M, Luttges J, Kloppel G, Jonat W and Nagasaki K: Expression of the tumor suppressor gene Maspin in human pancreatic cancers. Clin Cancer Res 7: 812-817, 2001

16. Sood AK, Fletcher MS, Gruman LM, Coffin JE, Jabbari S, Khalkhali-Ellis Z, Arbour N, Seftor EA and Hendrix MJ: The paradoxical expression of maspin in ovarian carcinoma. Clin Cancer Res 8: 2924-2932, 2002.

17. Hirai K, Koizumi K, Haraguchi S, Hirata T, Mikami I, Fukushima M, Yamagishi S, Kawashima T, Okada D, Shimizu K and Kawamoto M: Prognostic significance of the tumor suppressor gene maspin in non-small cell lung cancer. Ann Thorac Surg 79: 248-253, 2005.

18. Ito Y, Yoshida H, Tomoda C, Uruno T, Takamura Y, Miya A, Kobayashi K, Matsuzuka F, Matsuura N, Kuma K and Miyauchi A: Maspin expression is directly associated with biological aggressiveness of thyroid carcinoma. Thyroid 14: 13-18, 2004.

19. Sugimoto S, Maass N, Takimoto Y, Sato K, Minei S, Zhang M, Hoshikawa Y, Junemann KP, Jonat W and Nagasaki K: Expression and regulation of tumor suppressor gene maspin in human bladder cancer. Cancer Lett 203: 209-215, 2004.

20. Yu M, Zheng H, Tsuneyama K, Takahashi H, Nomoto K, Xu H and Takano Y: Paradoxical expression of maspin in gastric carcinomas: correlation with carcinogenesis and progression. Hum Pathol 38: 1248-1255, 2007.
21. Zheng H, Tsuneyama K, Cheng C, Takahashi H, Cui Z, Murai Y, Nomoto K and Takano Y: Maspin expression was involved in colorectal adenoma-adenocarcinoma sequence and liver metastasis of tumors. Anticancer Res 27: 259-265, 2007.

22. Zheng H, Takahashi H, Murai Y, Cui Z, Nomoto K, Niwa H, Tsuneyama K and Takano Y: Expressions of MMP-2, MMP-9 and VEGF are closely linked to growth, invasion, metastasis and angiogenesis of gastric carcinoma. Anticancer Res 26 3579-3583, 2006

23. Zheng HC, Sun JM, Li XH, Yang XF, Zhang YC and Xin Y: Role of PTEN and MMP-7 expression in growth, invasion, metastasis and angiogenesis of gastric carcinoma. Pathol Int 53: 659-666, 2003.

24. Zheng HC, Wang W, Xu XY, Xia P, Yu M, Sugiyama T and Takano Y: Up-regulated EMMPRIN/CD147 protein expression might play a role in colorectal carcinogenesis and its subsequent progression without an alteration of its glycosylation and mRNA level. J Cancer Res Clin Oncol 137: 585-596, 2011.

25. Zheng HC, Takahashi H, Murai Y, Cui ZG, Nomoto K, Miwa S, Tsuneyama K and Takano Y: Upregulated EMMPRIN/ CD147 might contribute to growth and angiogenesis of gastric carcinoma: a good marker for local invasion and prognosis. Br J Cancer 95: 1371-1378, 2006.

26. Li X, Zheng H, Hara T, Takahashi H, Masuda S, Wang Z, Yang X, Guan Y and Takano Y: Aberrant expression of cortactin and fascin are effective markers for pathogenesis, invasion, metastasis and prognosis of gastric carcinomas. Int J Oncol 33: 69-79, 2008.

27. Zheng HC, Zheng YS, Li XH, Takahashi H, Hara T, Masuda S, Yang XH, Guan YF and Takano Y: Arp2/3 overexpression contributed to pathogenesis, growth and invasion of gastric carcinoma. Anticancer Res 28: 2225-2232, 2008.

28. Takahashi H, Wang JP, Zheng HC, Masuda S and Takano Y: Overexpression of GRP78 and GRP94 is involved in colorectal carcinogenesis. Histol Histopathol 26: 663-671, 2011.

29. Zheng HC, Takahashi H, Li XH, Hara T, Masuda S, Guan YF and Takano Y: Overexpression of GRP78 and GRP94 are markers for aggressive behavior and poor prognosis in gastric carcinomas. Hum Pathol 39: 1042-1049, 2008

30. Zheng HC, Xu XY, Xia P, Yu M, Takahashi H and Takano Y: Involvement of inactive GSK3beta overexpression in tumorigenesis and progression of gastric carcinomas. Hum Pathol 41: 1255-1264, 2010

31. Zheng HC, Tsuneyama K, Takahashi H, Miwa S, Sugiyama T, Popivanova BK, Fujii C, Nomoto K, Mukaida N and Takano Y: Aberrant Pim-3 expression is involved in gastric adenoma adenocarcinoma sequence and cancer progression. J Cancer Res Clin Oncol 134: 481-488, 2008.

32. Li XH, Zheng HC, Wang ZG, Takahashi H, Yang XH, Guan YF and Takano Y: The clinicopathological and prognostic significance of MUC-1 expression in Japanese gastric carcinomas: an immunohistochemical study of tissue microarrays. Anticancer Res 28: 1061-1067, 2008

33. Zheng H, Takahashi H, Nakajima T, Murai Y, Cui Z, Nomoto K, Tsuneyama K and Takano Y: MUC6 down-regulation correlates with gastric carcinoma progression and a poor prognosis: an immunohistochemical study with tissue microarrays. J Cancer Res Clin Oncol 132: 817-823, 2006.

34. Zheng HC, Xu XY, Yu M, Takahashi H, Masuda S and Takano Y: The role of Reg IV gene and its encoding product in gastric carcinogenesis. Hum Pathol 41: 59-69, 2010.

35. Zheng HC, Sugawara A, Okamoto H, Takasawa S, Takahashi H, Masuda S and Takano Y: Expression profile of the REG gene family in colorectal carcinoma. J Histochem Cytochem 59: 106-115, 2011.

36. Zheng H, Takahashi H, Murai Y, Cui Z, Nomoto K, Miwa S, Tsuneyama $\mathrm{K}$ and Takano Y: Pathobiological characteristics of intestinal and diffuse-type gastric carcinoma in Japan: an immunostaining study on the tissue microarray. J Clin Pathol 60: 273-277, 2007.

37. Zheng HC, Li XH, Hara T, Masuda S, Yang XH, Guan YF and Takano Y: Mixed-type gastric carcinomas exhibit more aggressive features and indicate the histogenesis of carcinomas. Virchows Arch 452: 525-534, 2008.

38. Zheng HC, Zheng YS, Xia P, Xu XY, Xing YN, Takahashi H, Guan YF and Takano Y: The pathobiological behaviors and prognosis associated with Japanese gastric adenocarcinomas of pure WHO histological subtypes. Histol Histopathol 25: 445-452, 2010 